\title{
Farmacovigilância em Portugal: Atividade da Unidade Regional do Centro
}

\author{
Pharmacovigilance in Portugal: Activity of the Central Pharmacovigilance \\ Unit
}

Francisco BATEL-MARQUES ${ }^{1,2}$, Diogo MENDES ${ }^{1,2}$, Carlos ALVES ${ }^{1,2}$, Ana PENEDONES ${ }^{1}$, Patricia DIAS PI,3,4 $^{1,3}$ Angelina MARTINS ${ }^{1,3}$, Luiz Miguel SANTIAGO ${ }^{1,5}$, Carlos FONTES-RIBEIRO ${ }^{1,4}$, Margarida CARAMONA ${ }^{1,2}$, Tice MACEDO $^{1,4}$ Acta Med Port 2015 Mar-Apr;28(2):222-232

\section{RESUMO}

Introdução: Caracterizar as notificações espontâneas de eventos adversos a medicamentos recebidas pela Unidade de Farmacovigilância do Centro.

Material e Métodos: Consideraram-se todas as notificações reportadas entre 01/2001 e 12/2013. Estimaram-se taxas de notificação anuais. Os casos foram caracterizados quanto à gravidade, conhecimento prévio, causalidade imputada, origem e grupo profissional do notificador, tipo de evento adverso e grupos farmacoterapêuticos onde se incluem os medicamentos suspeitos com maior prevalência de notificação.

Resultados: A Unidade recebeu 2408 notificações, que continham 5749 eventos adversos. No ano de 2013 foi registada uma taxa de notificação de 171 notificações/milhão de habitantes. Do total de notificações, $55 \%$ foram classificadas como graves. Das notificações com causalidade imputada, $90 \%$ tinham uma relação pelo menos possível com o medicamento suspeito. Os medicamentos que originaram maior número de notificações foram os anti-infeciosos para uso sistémico $(n=809 ; 33 \%)$, e os eventos adversos mais frequentemente notificados foram as "Afeções dos tecidos cutâneos e subcutâneos" ( $n=1139 ; 20 \%)$. Registaram-se 154 (6,4\%) casos de risco de vida e/ou morte e $88(3,6 \%)$ continham pelo menos um evento adverso classificado simultaneamente como grave, desconhecido e definitivo ou provável.

Discussão: Os resultados deste estudo são consistentes com os de outros estudos, designadamente no que diz respeito à gravidade, aos grupos farmacoterapêuticos onde se incluem os medicamentos suspeitos e aos tipos de eventos adversos reportados.

Conclusão: Ao longo do período avaliado, a UFC solidificou a sua atividade, tendo verificado um crescimento da taxa de notificação em geral e um aumento da notificação de reações adversas graves e desconhecidas.

Palavras-chave: Farmacovigilância; Portugal; Sistemas de Notificação de Reações Adversas a Medicamentos.

ABSTRACT

Introduction: The aim of this study was to characterize the spontaneous reports of adverse events that were received by the Central Portugal Regional Pharmacovigilance Unit.

Material and Methods: Spontaneous reports received between 01/2001 and 12/2013 were considered. The annual reporting ratios were estimated. The cases were characterized according to their seriousness, previous description, causality assessment, origin and professional group of the reporter, type of adverse event and pharmacotherapeutic groups of the suspected drugs most frequently reported.

Results: The Pharmacovigilance Unit received 2408 reports that contained 5749 adverse events. In 2013 , the reporting rate was estimated at 171 reports per million inhabitants. Fifty-five percent of the reports were assessed as serious. Ninety percent of the cases were assessed as being at least possibly related with the suspected drug. The suspected drugs most frequently reported were antiinfectives for systemic use $(n=809,33 \%)$. The most frequently reported adverse events were "Skin and subcutaneous tissue disorders" $(n=1139,20 \%)$. There were $154(6.4 \%)$ reports resulting in life-threatening situations and/or death, and $88(3.6 \%)$ containing at least one adverse event assessed as serious, unknown and certain or probable.

Discussion: The present results are in line with those found in other studies, namely the seriousness and type of the adverse events and the pharmacotherapeutic groups of the most frequently reported suspected drugs.

Conclusion: In the last years, the Central Portugal Regional Pharmacovigilance Unit has registered a growth in the reporting rate in general, as well as an increase in the reporting of unknown and serious adverse drug reactions.

Keywords: Adverse Drug Reaction Reporting Systems; Pharmacovigilance; Portugal.

\section{INTRODUÇÃO}

Um evento adverso é definido como qualquer efeito deletério e indesejado que ocorre durante a utilização de um medicamento. ${ }^{1}$ Estes eventos podem desenvolver-se quando o medicamento é utilizado de acordo com os termos da sua autorização de introdução no mercado, podem resultar de situações de abuso ou má utilização, erros terapêuti- cos ou utilização off label (fora das indicações terapêuticas aprovadas). Situações de falta de eficácia do tratamento são também abrangidas pela definição de evento adverso e devem ser notificadas às autoridades reguladoras. O termo "reação adversa" aplica-se quando se suspeita de uma relação causal entre a toma do medicamento e a

1. Unidade de Farmacovigilância. Centro AIBILI - Associação para Investigação Biomédica e Inovação em Luz e Imagem. Coimbra. Portugal.

2. Faculdade de Farmácia. Universidade de Coimbra. Coimbra. Portugal.

3. Centro Hospitalar e Universitário de Coimbra. Coimbra. Portugal.

4. Faculdade de Medicina. Universidade de Coimbra. Coimbra. Portugal.

5. Faculdade de Ciências da Saúde. Universidade da Beira Interior. Covilhã. Portugal.

Recebido: 31 de Julho de 2014 - Aceite: 16 de Fevereiro de 2015 | Copyright @ Ordem dos Médicos 2015 
ocorrência do evento adverso, i.e., quando esta associação é considerada pelo menos possível pelo notificador ou por um método de imputação de causalidade. ${ }^{2}$

A iatrogenia medicamentosa tem um impacto significativo na saúde pública, sendo responsável por uma considerável morbi/mortalidade. ${ }^{3}$ Vários estudos dedicaram-se a avaliar a incidência de iatrogenia medicamentosa. Em doentes que recebem cuidados de saúde em ambulatório, estima-se que a incidência de eventos adversos varie entre 4 e 91/1000 pessoas-mês. ${ }^{4}$ Cerca de $5 \%$ das admissões hospitalares deve-se a iatrogenia medicamentosa. ${ }^{5-7} \mathrm{~A}$ incidência de eventos adversos varia de acordo com a faixa etária. A proporção de idosos que é admitida a nível hospitalar devido a iatrogenia medicamentosa é superior comparativamente à estimada para os outros doentes adultos e para as crianças $(10,7 \%$ vs. $6,3 \%$ vs. $4,1 \%$, respetivamente). ${ }^{6}$ Estima-se que as mortes suspeitas de estarem relacionadas com reações adversas a medicamentos ocorram em cerca de $0,3 \%$ a $5 \%$ dos doentes. ${ }^{8-11}$

A identificação da iatrogenia medicamentosa e a monitorização do seu impacto na população levou ao desenvolvimento de uma disciplina da Farmacoepidemiologia, a Farmacovigilância. ${ }^{12} \mathrm{O}$ objetivo da Farmacovigilância é avaliar os medicamentos e assegurar que os seus riscos não sejam superiores aos seus benefícios. ${ }^{1} \mathrm{O}$ Sistema Nacional de Farmacovigilância foi criado em 1992, de acordo com o Despacho Normativo n. ${ }^{\circ}$ 107/92, de 27 de Junho e descentralizado posteriormente, no ano 2000 , tendo sido criadas as Unidades Regionais de Farmacovigilância (URF) e distribuídas de acordo com as Administrações Regionais de Saúde. ${ }^{13}$ Desde a entrada em vigor da legislação europeia de Farmacovigilância mais recente, em 2012, para além dos profissionais de saúde, também os utentes estão autorizados a notificar suspeitas de reações adversas a medicamentos. ${ }^{14}$

A implementação das URF permitiu maior interação com os notificadores e uma maior divulgação do Sistema Nacional de Farmacovigilância, o que contribuiu para um aumento gradual do número de notificações de suspeitas de reações adversas ao longo dos anos. ${ }^{15}$ Para além da validação, processamento e avaliação dos casos notificados pelos profissionais de saúde e utentes, as URF também conduzem investigação na área da segurança do medicamento, contribuindo para um aumento do conhecimento na área da Farmacovigilância. ${ }^{16}$

O objetivo deste estudo é caracterizar as notificações espontâneas recebidas pela UFC quanto à origem, de acordo com o local de exercício da atividade dos notificadores, grupos farmacoterapêuticos dos medicamentos suspeitos, gravidade, conhecimento prévio e causalidade dos eventos adversos notificados.

\section{MATERIAL E MÉTODOS}

Este estudo, observacional, transversal e descritivo, considerou todas as notificações espontâneas de eventos adversos que foram reportadas à Unidade de Farmacovigilância do Centro entre 1 de janeiro de 2001 e 31 de dezem- bro de 2013. Foram incluídas as notificações espontâneas que verificaram os critérios de validação primária, designadamente a identificação do doente, medicamento suspeito, evento adverso e notificador.

O número de notificações/milhão habitante/ano foi calculado para os anos de 2011, 2012 e 2013. A população abrangida pela Administração Regional de Saúde do Centro de Portugal foi obtida a partir do Anuário Estatístico da Região Centro, tendo sido identificados 1719973 habitantes. ${ }^{17}$

As notificações espontâneas foram caracterizadas quanto à origem (hospital, ambulatório, farmácia ou outro) e ao grupo profissional do notificador (médico, farmacêutico, enfermeiro, outro profissional de saúde ou utente). A designação de "Ambulatório" foi utilizada para identificar a origem dos casos notificados a partir das instituições pertencentes à rede primária dos cuidados de saúde.

Cada notificação espontânea refere-se a um único caso (um doente), mas pode incluir um ou mais eventos adversos associados ao uso de um ou mais medicamentos suspeitos. Os doentes foram caracterizados demograficamente quanto ao género e à idade.

Os medicamentos suspeitos foram classificados de acordo com a Classificação ATC (Anatomical Therapeutic Chemical Code) da Organização Mundial da Saúde (OMS) no primeiro nível (grupo anatómico principal) e no terceiro nível (subgrupo farmacológico). De acordo com a classificação ATC da OMS, as substâncias ativas são divididas em diferentes grupos de acordo com o órgão ou sistema em que atuam e as suas propriedades terapêuticas, farmacológicas e químicas. ${ }^{18}$

Dado que uma notificação espontânea contém pelo menos um evento adverso, cada evento foi isolado e codificado de acordo com o dicionário MedDRA (Medical Dictionary for Regulatory Activities), versão 17.0 [Maintenance and Support Services Organization (MSSO) McLean, VA, USA], no Sistema de Classificação de Órgãos Primário (SOC) e no Termo Preferido (PT). ${ }^{19-21}$ A codificação MedDRA é uma terminologia médica específica e padronizada, desenvolvida pela Conferência Internacional de Harmonização, para facilitar a partilha de informação entre as autoridades reguladoras e a indústria farmacêutica sobre regulamentação de produtos médicos (medicamentos, dispositivos médicos) utilizados por seres humanos. É utilizada para registo, documentação e monitorização da segurança de produtos médicos nas fases de investigação e desenvolvimento e pós-comercialização. ${ }^{22}$

Os eventos adversos foram considerados graves se "resultaram em morte", "colocaram a vida em risco", "motivaram ou prolongaram a hospitalização", "motivaram incapacidade temporária e/ou definitiva", e/ou "originaram anomalias congénitas". Outros eventos adversos, que apesar de à data de ocorrência não terem colocado em risco a vida do doente ou resultado na sua hospitalização, mas que estiveram associados a consequências clínicas importantes e/ou exigiram a intervenção médica para reverter também foram considerados graves. ${ }^{23}$ Os eventos adversos foram 
considerados conhecidos se estivessem descritos no Resumo de Características do Medicamento suspeito. A relação causal entre a exposição ao medicamento suspeito e a ocorrência dos eventos adversos foi avaliada pelo ConseIho Científico da UFC de acordo com o método da introspeção global. Um evento adverso pode ser classificado como definitivo, provável, possível, improvável, condicional/não classificado ou não acessível/não classificável. ${ }^{24} \mathrm{O}$ método da introspeção global é um processo pelo qual um conjunto de peritos avalia a relação causal entre a exposição a um medicamento e a ocorrência de um evento adverso, tendo em conta os critérios de Bradford Hill: força, consistência, especificidade, temporalidade, gradiente biológico, plausibilidade, coerência, evidência experimental e analogia. ${ }^{25}$ Este método assemelha-se ao processo de diagnóstico clínico e depende dos conhecimentos e da experiência dos peritos. ${ }^{26} \mathrm{O}$ método da introspeção global tem um grau de correspondência moderado a elevado com os algoritmos decisionais para reações adversas mais provavelmente relacionadas com o medicamento suspeito. ${ }^{16}$

Foram realizadas análises com o objetivo de caracterizar dois subgrupos de notificações espontâneas de eventos adversos: os casos que colocaram em risco a vida e/ ou resultaram em morte do doente, independentemente da relação causal com o medicamento suspeito; e os casos que foram classificados simultaneamente como graves, desconhecidos e definitivos ou prováveis quanto à relação causal com os medicamentos suspeitos.

O software Microsoft Exce ${ }^{\circledR} 2010$ (Microsoft Corporation, Santa Rosa, CA, USA) foi utilizado para o tratamento estatístico dos dados.

\section{RESULTADOS}

Entre janeiro de 2001 e dezembro de 2013, a UFC recebeu 2408 notificações espontâneas de eventos adversos.

\section{Taxa de notificação}

Nos anos de 2011, 2012 e 2013 foram recebidas 101, 123 e 171 notificações/ milhão de habitante/ ano, respetivamente.

\section{Origem da notificação e tipo de notificador}

A distribuição das notificações espontâneas recebidas por origem de notificação e tipo de notificador apresenta-se na Tabela 1. Do total dos casos recebidos, 943 (40\%) tiveram origem no ambulatório, 778 (32\%) no hospital, 502 $(21 \%)$ na farmácia comunitária e $185(8 \%)$ noutros locais de trabalho ou, no caso dos utentes, na morada de residência. Os profissionais de saúde que mais notificaram foram os médicos $(1457 ; 61 \%)$, seguidos dos farmacêuticos $(693$; $29 \%$ ) e dos enfermeiros $(239 ; 10 \%)$. Os utentes notificaram 13 casos $(0,5 \%)$.

\section{Características demográficas dos doentes}

A maioria das notificações espontâneas referia-se a doentes do género feminino $(n=1594 ; 66 \%)$. A mediana das idades dos doentes era de 53 anos (mínimo 2 meses, máximo 96 anos) à data da ocorrência do evento adverso. A maioria dos doentes tinha idade compreendida entre os 15 e os 65 anos ( $n=1385 ; 58 \%)$. Duzentos e sessenta e nove doentes tinham idade inferior a 15 anos (11\%) e 683 doentes tinham idade superior a 65 anos (28\%). Não foi possível obter informação acerca da idade do doente em 72 casos.

\section{Medicamentos suspeitos}

Trinta casos recebidos na UFC (1\%) indicavam mais do que um medicamento suspeito. No total, foram considerados 2.448 medicamentos suspeitos. As classes ATC onde se incluem os medicamentos suspeitos mais frequentemente notificados encontram-se descritas na Tabela 2. De acordo com o primeiro nível ATC, os medicamentos que originaram maior número de notificações espontâneas foram os anti-infeciosos para uso sistémico ( $n=809 ; 33 \%$ ), e os que atuam no sistema musculoesquelético ( $n=322 ; 13 \%)$ e no sistema nervoso central ( $n=312 ; 13 \%)$.

Os medicamentos suspeitos incluídos no grupo das vacinas foram os mais frequentemente notificados na população com idades compreendidas entre os 0 e os 15 anos de idade $(n=191 ; 69 \%)$. Nos restantes grupos populacionais, os anti-inflamatórios não-esteroides foram os mais frequentemente notificados $(n=209 ; 10 \%)$.

Tabela 1 - Notificações espontâneas distribuídas de acordo com o notificador e origem de notificação

\begin{tabular}{|c|c|c|c|c|c|}
\hline \multirow{2}{*}{ Tipo de Notificador } & \multicolumn{5}{|c|}{ Origem de Notificação $§$} \\
\hline & Ambulatório & Farmácia & Hospital & Outro & Total \\
\hline Enfermeiro & 166 & 0 & 73 & 0 & 239 \\
\hline Farmacêutico & 0 & 499 & 175 & 19 & 693 \\
\hline Médico & 777 & 0 & 528 & 152 & 1457 \\
\hline Outro profissional de saúde & 0 & 3 & 2 & 1 & 6 \\
\hline Utente ${ }^{\pi}$ & 0 & 0 & 0 & 13 & 13 \\
\hline Total & 943 & 502 & 778 & 185 & 2408 \\
\hline
\end{tabular}

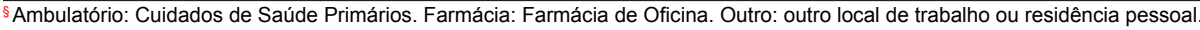

"Utentes só começaram a notificar a partir de Julho de 2012. 
Tabela 2 - Medicamentos suspeitos, classificados no $3 .^{\circ}$ nível ATC, mais frequentemente notificados (frequência $\geq 0,5 \%$ )

\begin{tabular}{|c|c|c|}
\hline Medicamentos suspeitos & $\mathbf{n}$ & $\%$ \\
\hline J07B Vacinas virais & 236 & $9,64 \%$ \\
\hline M01A Anti-inflamatórios e antirreumáticos não esteroides & 218 & $8,91 \%$ \\
\hline J07A Vacinas bacterianas & 173 & $7,07 \%$ \\
\hline N06A Antidepressivos & 102 & $4,17 \%$ \\
\hline L01X Outros agentes antineoplásicos & 97 & $3,96 \%$ \\
\hline J01C Antibacterianos beta-lactâmicos, penicilinas & 96 & $3,92 \%$ \\
\hline C10A Agentes modificadores dos lípidos, simples & 86 & $3,51 \%$ \\
\hline J01M Antibacterianos quinolonas & 82 & $3,35 \%$ \\
\hline L01C Alcalóides de plantas e outros produtos naturais & 70 & $2,86 \%$ \\
\hline J01D Outros antibacterianos beta-lactâmicos & 59 & $2,41 \%$ \\
\hline C09A Inibidores da Enzima de Conversão da Angiotensina (ECA), simples & 51 & $2,08 \%$ \\
\hline B01A Agentes antitrombóticos & 50 & $2,04 \%$ \\
\hline J01F Macrólidos, lincosamidas e estreptograminas & 47 & $1,92 \%$ \\
\hline N03A Antiepiléticos & 47 & $1,92 \%$ \\
\hline N02B Outros analgésicos e antipiréticos & 46 & $1,88 \%$ \\
\hline A02B Medicamentos para a úlcera péptica doença de refluxo gastro-esofágico & 43 & $1,76 \%$ \\
\hline M05B Medicamentos que afetam a estrutura e a mineralização do osso & 43 & $1,76 \%$ \\
\hline M04A Preparações antigotosas & 36 & $1,47 \%$ \\
\hline J01X Outros antibacterianos & 30 & $1,23 \%$ \\
\hline G03A Contraceptivos hormonais para uso sistémico & 29 & $1,18 \%$ \\
\hline J01E Sulfonamidas e trimetoprim & 27 & $1,10 \%$ \\
\hline C08C Bloqueadores seletivos dos canais de cálcio com efeitos principalmente vasculares & 26 & $1,06 \%$ \\
\hline L01B Antimetabolitos & 25 & $1,02 \%$ \\
\hline L04A Imunossupressores & 25 & $1,02 \%$ \\
\hline N02A Opióides & 23 & $0,94 \%$ \\
\hline N05A Antipsicóticos & 22 & $0,90 \%$ \\
\hline L02B Antagonistas hormonais e agentes relacionados & 20 & $0,82 \%$ \\
\hline V08A Meios de contraste para Raio-X, iodados & 20 & $0,82 \%$ \\
\hline N05B Ansiolíticos & 19 & $0,78 \%$ \\
\hline N05C Hipnóticos e sedativos & 17 & $0,69 \%$ \\
\hline C09B Inibidores da ECA, combinações & 16 & $0,65 \%$ \\
\hline C09C Antagonistas da angiotensina II, simples & 16 & $0,65 \%$ \\
\hline L03A Imunoestimulantes & 16 & $0,65 \%$ \\
\hline R05C Expetorantes, excl. combinações com supressores da tosse & 16 & $0,65 \%$ \\
\hline J07C Vacinas bacterianas e virais, combinações & 15 & $0,61 \%$ \\
\hline R06A Antihistamínicos para uso sistémico & 14 & $0,57 \%$ \\
\hline G04C Medicamentos usados na hipertrofia benigna da próstata & 13 & $0,53 \%$ \\
\hline H02A Corticosteroides para uso sistémico, simples & 13 & $0,53 \%$ \\
\hline M03B Relaxantes musculares, agentes com atuação central & 13 & $0,53 \%$ \\
\hline Outros & 448 & $18,30 \%$ \\
\hline TOTAL & 2448 & $100,00 \%$ \\
\hline
\end{tabular}




\section{Eventos adversos}

Dos 2408 casos recebidos na UFC, 1540 (64\%) reportaram mais do que um evento adverso. No total, foram reportados 5749 eventos adversos. Os eventos adversos mais frequentemente notificados, codificados de acordo com o PT e agrupados por SOC, encontram-se descritos na Tabela 3. O SOC com maior frequência de eventos adversos foi "Afeções dos tecidos cutâneos e subcutâneos" $(n=1139 ; 20 \%$ ), seguido de "Perturbações gerais e alterações no local de administração" ( $n=1084 ; 19 \%)$ e "Doenças gastrointestinais" ( $n=1034 ; 18 \%$ ). Estes três grupos totalizaram $57 \%$ dos eventos adversos reportados.

Os eventos adversos mais frequentemente reportados na população com idades compreendidas entre os 0 e os 15 anos estiveram relacionados com a vacinação, designadamente "vacinação ineficaz", "aumento de volume da glândula parótida" e "pirexia". O "prurido", a "diarreia" e o "vómito" foram os eventos adversos mais frequentes no subgrupo populacional com idade $\geq 65$ anos.

\section{Eventos adversos que resultaram em risco de vida e/ ou morte}

Das 2408 notificações espontâneas, 1316 (55\%) foram classificadas como graves. Das notificações espontâneas graves, $154(6,4 \%)$ resultaram em risco de vida e/ou morte. Dessas, $118(76,6 \%)$ foram notificados por médicos, 20 (13\%) por farmacêuticos, $15(9,7 \%)$ por enfermeiros e 1 $(0,6 \%)$ por um utente (embolia pulmonar). A maioria das notificações espontâneas referia-se a doentes do sexo feminino $(n=97 ; 63 \%)$. A mediana das idades dos doentes era de 56 anos (mínimo 2 meses, máximo 93 anos) à data da ocorrência do evento adverso. As classes ATC dos medicamentos suspeitos e os eventos adversos mais frequentemente reportados em casos que colocaram a vida em risco e/ou resultaram em morte dos doentes apresentam-se na Tabela 4 e Tabela 5, respetivamente. Os medicamentos utilizados no tratamento de doenças oncológicas, que inclui os agentes antineoplásicos $(n=21 ; 13 \%)$, os alcaloides ( $=10 ; 6 \%)$, os antimetabolitos $(n=4 ; 3 \%)$ e os agentes alquilantes $(n=4 ; 3 \%)$, foram os mais frequentemente associados a estes casos. Os quadros de anafilaxia, que podem incluir "reação anafilática" ( $n=21 ; 5 \%)$, "choque anafilático" ( $n=16 ; 4 \%)$, "dispneia" ( $n=15 ; 4 \%)$, "hipotensão" $(n=11$; $3 \%)$ e "edema da laringe" ( $n=9 ; 2 \%)$ foram os eventos mais frequentemente associados a casos que colocaram a vida em risco e/ou resultaram em morte.

\section{Eventos adversos graves, desconhecidos e definitivos ou prováveis}

A UFC recebeu 88 notificações espontâneas que continham pelo menos um evento adverso classificado como grave, desconhecido e definitivo ou provável. Os medicamentos suspeitos reportados apresentam-se na Tabela 6 . Os anti-infeciosos para uso sistémico $(n=27 ; 30 \%)$, os antineoplásicos $(n=21 ; 23 \%)$ e os medicamentos que atuam no sistema musculoesquelético $(n=12 ; 13 \%)$ foram os medicamentos suspeitos mais prevalentes.

\section{DISCUSSÃO}

A notificação espontânea é uma metodologia de farmacovigilância que permite identificar eventos adversos, abrangendo todos os medicamentos comercializados e todos os doentes, particularmente os que integram populações sub-representadas nos ensaios clínicos, como os idosos, as grávidas e as crianças. A notificação espontânea também permite detetar eventos adversos raros e/ou de longo tempo de latência. ${ }^{27-29}$

A taxa de notificação é um dos indicadores de desempenho da atividade das URF. Comparativamente aos resultados obtidos num estudo anterior verificou-se um aumento do número de notificações espontâneas por milhão de habitantes por ano recebidas pela UFC ao longo dos últimos 3 anos (máximo de 171 casos/milhão de habitantes/ano em 2013). ${ }^{24}$ Este resultado aproxima-se da recomendação da Organização Mundial de Saúde ( $\geq 200$ casos/milhão de habitantes/ano). ${ }^{24,30}$

Mais de metade dos casos (55\%) recebidos pela UFC foram classificados como graves. Este resultado é consistente com a proporção de iatrogenia grave identificada noutras URFs em Portugal, e também com os resultados de estudos que avaliaram sistemas de Farmacovigilância de outros países Europeus. ${ }^{15,31,32}$ Todos os eventos adversos devem ser notificados às autoridades reguladoras. No entanto, embora não haja evidência que o demonstre, pode suspeitar-se que os profissionais de saúde estão particularmente sensibilizados a reportar apenas eventos adversos graves, uma vez que estes estão associados a um aumento da morbilidade e dos custos em saúde..$^{32}$

A maioria das notificações espontâneas recebidas pela UFC durante o seu período de atividade foi reportada por médicos, estando em linha com os resultados descritos noutros estudos. ${ }^{15,31-34}$ Seguem-se os farmacêuticos e depois os enfermeiros. Face aos resultados apresentados num estudo anterior, com um delineamento idêntico e que contemplou as notificações espontâneas reportadas à UFC entre 2001 e 2011, a proporção de casos notificados por enfermeiros registou um aumentou considerável. ${ }^{24}$ Isto deve-se, em parte, às ações de divulgação realizadas pela UFC junto destes profissionais de saúde, com o objetivo de aumentar o conhecimento acerca da importância em documentar iatrogenia medicamentosa e dos procedimentos de notificação. ${ }^{35} \mathrm{Na}$ prática profissional, os enfermeiros têm um contacto próximo com os doentes. ${ }^{35}$ Estes profissionais são responsáveis pela administração e monitorização da terapêutica, particularmente nos idosos, estando numa posição privilegiada para identificar iatrogenia medicamentosa. ${ }^{35-39}$

Apesar da nova legislação de Farmacovigilância permitir que tanto os profissionais de saúde como os utentes possam notificar suspeitas de reações adversas, a contribuição destes últimos tem sido residual. ${ }^{40}$ Os utentes já estavam autorizados a notificar suspeitas de reações adversas há alguns anos noutros países, por exemplo na Dinamarca, na Holanda ou no Reino Unido. ${ }^{41,42}$ Apesar dos primeiros estudos dedicados a avaliar o contributo da 
Tabela 3 - Eventos adversos, classificados no PT do MedDRA, mais frequentemente notificados (frequência $\geq 0,5 \%$ )

\begin{tabular}{|c|c|c|}
\hline Eventos adversos & $\mathbf{n}$ & $\%$ \\
\hline Doenças Cardíacas & 104 & $1,81 \%$ \\
\hline Taquicardia & 53 & $0,92 \%$ \\
\hline Outros & 51 & $0,89 \%$ \\
\hline Doenças Gastrointestinais & 1034 & $17,99 \%$ \\
\hline Vómito & 164 & $2,85 \%$ \\
\hline Náusea & 153 & $2,66 \%$ \\
\hline Aumento de volume da glândula parótida & 137 & $2,38 \%$ \\
\hline Diarreia & 134 & $2,33 \%$ \\
\hline Dor abdominal & 111 & $1,93 \%$ \\
\hline Dispepsia & 42 & $0,73 \%$ \\
\hline Outros & 293 & $5,10 \%$ \\
\hline Perturbações gerais e alterações no local de administração & 1084 & $18,86 \%$ \\
\hline Pirexia & 197 & $3,43 \%$ \\
\hline Mal-estar geral & 81 & $1,41 \%$ \\
\hline Edema facial & 64 & $1,11 \%$ \\
\hline Inflamação no local de injeção & 60 & $1,04 \%$ \\
\hline Eritema no local de injeção & 54 & $0,94 \%$ \\
\hline Edema no local de injeção & 50 & $0,87 \%$ \\
\hline Edema periférico & 48 & $0,83 \%$ \\
\hline Astenia & 46 & $0,80 \%$ \\
\hline Edema & 43 & $0,75 \%$ \\
\hline Fármaco ineficaz & 39 & $0,68 \%$ \\
\hline Dor no local de injeção & 36 & $0,63 \%$ \\
\hline Calor no local de injeção & 35 & $0,61 \%$ \\
\hline Sensação de calor & 30 & $0,52 \%$ \\
\hline Outros & 218 & $3,79 \%$ \\
\hline Doenças do sistema imunitário & 82 & $1,43 \%$ \\
\hline Reação anafilática & 30 & $0,52 \%$ \\
\hline Outros & 52 & $0,90 \%$ \\
\hline Infeções e Infestações & 151 & $2,63 \%$ \\
\hline Papeira & 74 & $1,29 \%$ \\
\hline Outros & 77 & $1,34 \%$ \\
\hline Complicações de intervenções relacionadas com lesões e intoxicações & 161 & $2,80 \%$ \\
\hline Vacinação ineficaz & 140 & $2,44 \%$ \\
\hline Outros & 21 & $0,37 \%$ \\
\hline Afeções musculoesqueléticas e dos tecidos conjuntivos & 215 & $3,74 \%$ \\
\hline Mialgia & 56 & $0,97 \%$ \\
\hline Dorsalgia & 45 & $0,78 \%$ \\
\hline Outros & 114 & $1,98 \%$ \\
\hline
\end{tabular}


Tabela 3 - Eventos adversos, classificados no PT do MedDRA, mais frequentemente notificados (frequência $\geq 0,5 \%$ )

\begin{tabular}{|c|c|c|}
\hline Eventos adversos & $\mathbf{n}$ & $\%$ \\
\hline Doenças do sistema nervoso & 601 & $10,45 \%$ \\
\hline Cefaleia & 132 & $2,30 \%$ \\
\hline Tonturas & 120 & $2,09 \%$ \\
\hline Tremor & 62 & $1,08 \%$ \\
\hline Sonolência & 35 & $0,61 \%$ \\
\hline Parestesia & 30 & $0,52 \%$ \\
\hline Outros & 222 & $3,86 \%$ \\
\hline Perturbações do foro psiquiátrico & 122 & $2,12 \%$ \\
\hline Insónia & 42 & $0,73 \%$ \\
\hline Outros & 80 & $1,39 \%$ \\
\hline Doenças respiratórias, torácicas e do mediastino & 282 & $4,91 \%$ \\
\hline Dispneia & 90 & $1,57 \%$ \\
\hline Tosse & 56 & $0,97 \%$ \\
\hline Outros & 136 & $2,37 \%$ \\
\hline Afeções dos tecidos cutâneos e subcutâneos & 1139 & $19,81 \%$ \\
\hline Prurido & 196 & $3,41 \%$ \\
\hline Erupção cutânea & 136 & $2,37 \%$ \\
\hline Eritema & 101 & $1,76 \%$ \\
\hline Erupção máculopapulosa & 85 & $1,48 \%$ \\
\hline Urticária & 82 & $1,43 \%$ \\
\hline Erupção eritematosa & 71 & $1,23 \%$ \\
\hline Hiperidrose & 43 & $0,75 \%$ \\
\hline Erupção cutânea generalizada & 43 & $0,75 \%$ \\
\hline Prurido generalizado & 39 & $0,68 \%$ \\
\hline Angioedema & 36 & $0,63 \%$ \\
\hline Outros & 307 & $5,34 \%$ \\
\hline Vasculopatias & 193 & $3,36 \%$ \\
\hline Afrontamento & 91 & $1,58 \%$ \\
\hline Hipotensão & 37 & $0,64 \%$ \\
\hline Hipertensão & 29 & $0,50 \%$ \\
\hline Outros & 36 & $0,63 \%$ \\
\hline Outros SOC & 581 & $10,11 \%$ \\
\hline Total & 5749 & $100,00 \%$ \\
\hline
\end{tabular}

notificação por utentes para a Farmacovigilância terem sugerido que a sensibilidade da notificação de suspeitas de reações adversas era baixa, a evidência mais recente considera que a contribuição dos utentes para a identificação da iatrogenia medicamentosa é valiosa, uma vez que estes poderão reportar suspeitas de reações adversas até então desconhecidas. ${ }^{41-43}$ Dado que os profissionais de saúde e os utentes fornecem diferentes perspetivas no que diz respeito à notificação de suspeitas de reações adversas, em que os primeiros estão mais focados na causalidade e os últimos na severidade e impacto sobre a qualidade de vida, é importante receber notificações de ambos os grupos para avaliar melhor a natureza dos eventos. ${ }^{44}$

A consulta em ambulatório foi o local de origem mais frequentemente indicado nas notificações espontâneas, seguido dos hospitais e da farmácia comunitária. O hospital é apontado noutros estudos como a origem mais frequente de notificações espontâneas. ${ }^{32-34} \mathrm{~A}$ incidência de iatrogenia 
medicamentosa em doentes hospitalizados é considerada elevada. ${ }^{9}$ No entanto, é reconhecido que a maioria dos eventos adversos que ocorrem a nível hospitalar não é reportada às autoridades reguladoras. ${ }^{45}$ Algumas das razões apontadas como possíveis causas da subnotificação nos hospitais são a falta de organização do sistema de Farmacovigilância hospitalar, falta de tempo dos profissionais de saúde ou problemas com a confidencialidade da informação. ${ }^{46}$

Os medicamentos suspeitos mais frequentemente notificados, nos casos recebidos pela UFC, foram os anti-infeciosos de uso sistémico, onde se inserem as vacinas e os antibióticos, seguidos dos medicamentos que atuam no sistema musculosquelético, na sua maioria anti-inflamatórios não-esteroides, e dos medicamentos que atuam no sistema nervoso, principalmente antidepressivos, antipsicóticos e ansiolíticos. Estes resultados estão em linha com os dados nacionais. ${ }^{15}$ Deve ressalvar-se que, nos últimos dois anos, a UFC recebeu um número considerável de notificações espontâneas cujo medicamento suspeito era a vacina contra a parotidite epidémica, uma vez que se registou um surto desta doença na região centro de Portugal. ${ }^{47}$ No entanto, apesar deste acréscimo de casos em particular, os resultados da UFC estão em linha com os registados noutros estudos. ${ }^{32,48}$ Os medicamentos incluídos nestas classes terapêuticas são dos mais utilizados na prática clínica, o que pode explicar que a proporção de casos recebidos na UFC em que eles são reportados como suspeitos seja elevada. ${ }^{49}$ Existem medicamentos cuja relação benefício/ risco é mais desfavorável comparativamente aos medicamentos suspeitos mais frequentemente notificados neste estudo, e que estão indicados para tratar patologias mais graves, tais como o cancro ou as doenças autoimunes. Assim, quando se limitou a análise aos casos graves em que houve risco para a vida do doente ou que resultaram em morte, os medicamentos utilizados no tratamento de doenças oncológicas foram os mais frequentemente notificados. Estes resultados eram de alguma forma expectáveis tendo em conta que, geralmente, a tolerância ao risco da ocorrência de eventos adversos graves tende a ser mais elevada no caso de o medicamento ser utilizado no tratamento de doenças severas. ${ }^{50} \mathrm{O}$ facto destes medicamentos serem administrados em meio hospitalar sob a supervisão atenta de profissionais de saúde permite a deteção de eventos adversos graves numa fase muito inicial e a atuação imediata com vista à reversão dos mesmos. Apesar da existência e da aplicação de procedimentos que visam prevenir a ocorrência de eventos adversos associados à terapêutica antineoplásica, incluindo a administração prévia de corticoides e anti-histamínicos, as reações do tipo anafilático continuam a manifestar-se em doentes oncológicos. ${ }^{51} \mathrm{~A}$ eficácia das medidas preventivas merece uma investigação futura mais aprofundada.

Tabela 4 - Medicamentos suspeitos notificados em casos de risco de vida e/ou morte

\begin{tabular}{|c|c|c|}
\hline Medicamentos suspeitos & $\mathbf{n}$ & $\%$ \\
\hline L01X Outros medicamentos antineoplásicos & 21 & $13,29 \%$ \\
\hline M01A Anti-inflamatórios e antirreumáticos não esteroides & 17 & $10,76 \%$ \\
\hline L01C Alcalóides de plantas e outros produtos naturais & 10 & $6,33 \%$ \\
\hline N02B Outros analgésicos e antipitéticos & 7 & $4,43 \%$ \\
\hline B01A Agentes antitrombóticos & 6 & $3,80 \%$ \\
\hline J01D Outros antibacterianos beta-lactâmicos & 5 & $3,16 \%$ \\
\hline M04A Preparações antigotosas & 5 & $3,16 \%$ \\
\hline J01E Sulfonamidas e trimetoprim & 5 & $3,16 \%$ \\
\hline J07B Vacinas virais & 5 & $3,16 \%$ \\
\hline V08A Meios de contraste para Raio-X, iodados & 4 & $2,53 \%$ \\
\hline L01B Antimetabolitos & 4 & $2,53 \%$ \\
\hline A10B Outros medicamentos hipoglicemiantes, excl. insulinas & 4 & $2,53 \%$ \\
\hline L04A Imunossupressores & 4 & $2,53 \%$ \\
\hline L01A Agentes alquilantes & 4 & $2,53 \%$ \\
\hline Outros & 58 & $36,48 \%$ \\
\hline Total & 159 & $100,00 \%$ \\
\hline
\end{tabular}


Tabela 5 - Eventos adversos notificados em casos de risco de vida e/ou morte

\begin{tabular}{lcc}
\hline Eventos adversos & $\mathbf{n}$ & $\%$ \\
\hline Reação anafilática & 21 & $5,33 \%$ \\
Choque anafilático & 16 & $4,06 \%$ \\
Dispneia & 15 & $3,81 \%$ \\
Hipotensão & 11 & $2,79 \%$ \\
Edema da laringe & 9 & $2,28 \%$ \\
Vómito & 9 & $2,28 \%$ \\
Eritema & 9 & $2,28 \%$ \\
Angioedema & 8 & $2,03 \%$ \\
Urticária & 7 & $1,78 \%$ \\
Prurido & 7 & $1,78 \%$ \\
Outros & 282 & $71,57 \%$ \\
Total & 394 & $100,00 \%$ \\
\hline
\end{tabular}

Tal como observado noutros estudos, as reações que envolveram a pele e os tecidos moles, o sistema gastrointestinal e os distúrbios gerais e reações que ocorreram no local de administração, foram as mais notificadas pelos profissionais de saúde. ${ }^{15,32,52} \mathrm{O}$ facto dos eventos adversos mais frequentes terem sido os dermatológicos é de certa forma expectável, uma vez que estes são facilmente identificados pelos doentes e profissionais de saúde. ${ }^{32}$ Distúrbios como a diarreia, náuseas, vómitos, mal-estar generalizado, febre e reações no local de administração, no caso dos injetáveis, são dos eventos adversos que ocorrem, presumivelmente, com maior frequência na prática clínica, daí constituírem uma proporção considerável da iatrogenia recebida na UFC.

Oitenta e oito casos notificados continham pelo menos um evento adverso que foi classificado simultaneamente como grave, desconhecido e definitivo ou provável. Os dois níveis de causalidade selecionados significam uma maior força de associação entre a exposição a um medicamento suspeito e a ocorrência de eventos adversos, que resultaram em consequências clínicas graves e cuja possibilidade de ocorrência era desconhecida. Estes casos merecem especial atenção por representarem o novo conhecimento produzido decorrente da atividade da UFC. Comparativamente aos resultados obtidos num estudo anterior, o número absoluto de casos com estas características mais do que duplicou em cerca de dois anos e meio. ${ }^{24}$ Este resultado sugere a importância e a relevância crescente da atividade da UFC e dos notificadores da região Centro na produção de novo conhecimento acerca da segurança de medicamentos.

Tabela 6 - Medicamentos suspeitos notificados nos casos graves, desconhecidos e definitivos ou prováveis

\begin{tabular}{|c|c|c|}
\hline Medicamentos suspeitos & $\mathbf{n}$ & $\%$ \\
\hline J Anti-infecciosos de uso sistémico & 27 & $29,67 \%$ \\
\hline L Agentes antineoplásicos e imunomoduladores & 21 & $23,08 \%$ \\
\hline M Sistema musculo-esquelético & 12 & $13,19 \%$ \\
\hline N Sistema nervoso & 8 & $8,79 \%$ \\
\hline C Sistema Cardiovascular & 5 & $5,49 \%$ \\
\hline V Vários & 4 & $4,40 \%$ \\
\hline A Trato gastrointestinal e metabolismo & 4 & $4,40 \%$ \\
\hline B Sangue e órgãos hematopoiéticos & 3 & $3,30 \%$ \\
\hline R Aparelho respiratório & 2 & $2,20 \%$ \\
\hline S Órgãos dos sentidos & 2 & $2,20 \%$ \\
\hline G Sistema genito-urinário e órgãos sexuais & 1 & $1,10 \%$ \\
\hline D Fármacos usados em dermatologia & 1 & $1,10 \%$ \\
\hline H Fármacos hormonais sistémicos, com exclusão das hormonas sexuais e insulinas & 1 & $1,10 \%$ \\
\hline Total & 91 & $100,00 \%$ \\
\hline
\end{tabular}


A evidência sobre os riscos é maioritariamente obtida a partir de dados pós-comercialização, incluindo a notificação espontânea, relatos de casos e estudos observacionais. ${ }^{53} \mathrm{~A}$ maioria das decisões regulamentares levadas a cabo pelas autoridades reguladoras acerca da segurança de medicamentos, incluindo a retirada de medicamentos do mercado e a geração de alertas de segurança, tem sido maioritariamente baseada em notificação espontânea. ${ }^{49,53-58}$ Mais de metade dos alertas de segurança gerados pelas autoridades reguladoras entre 2010 e 2012 tiveram por base notificações espontâneas, dos quais $20 \%$ exclusivamente através desta fonte de evidência. ${ }^{49}$ Estes resultados sugerem a importância da notificação espontânea na geração de novo conhecimento acerca da segurança de medicamentos. No entanto, a dificuldade em reconhecer, diagnosticar e estabelecer uma relação causal entre um evento adverso e a utilização de um medicamento constituem barreiras à notificação espontânea. ${ }^{59}$ A subnotificação por parte dos profissionais de saúde é um problema transversal a todos os sistemas de Farmacovigilância. ${ }^{19,24,35,60}$

\section{REFERÊNCIAS}

1. European Medicines Agency. New EU pharmacovigilance legislation - Key concepts. EMA/186974/2012; 2013; [consultado 2014 Jul 22]. Disponivel em: http://www.ema.europa.eu/docs/en_GB/document library/Other/2012/07/WC500129593.pdf.

2. Lindquist $M$. The need for definitions in pharmacovigilance. Drug Saf. 2007;30:825-30.

3. Gandhi TK, Weingart SN, Borus J, Seger AC, Peterson J, Burdick E, et al. Adverse events in ambulatory care. N Engl J Med. 2003;348:155664.

4. Thomsen LA, Winterstein AG, Søndergaard B, Haugbølle LS, Melander A. Systematic review of the incidence and characteristics of preventable adverse drug events in ambulatory care. Ann Pharmacother. 2007;41:1411-26.

5. Beijer HJ, de Blaey CJ. Hospitalisations caused by adverse drug reactions (ADR): a meta-analysis of observational studies. Pharm World Sci. 2002;24:46-54.

6. Kongkaew C, Noyce PR, Ashcroft DM. Hospital admissions associated with adverse drug reactions: a systematic review of prospective observational studies. Ann Pharmacother. 2008;42:1017-25.

7. Brvar M, Fokter N, Bunc M, Mozina M. The frequency of adverse drug reaction related admissions according to method of detection, admission urgency and medical department specialty. BMC Clin Pharmacol. 2009;9:8.

8. van der Hooft CS, Dieleman JP, Siemes C, Aarnoudse AJ, Verhamme $\mathrm{KM}$, Stricker BH, et al. Adverse drug reaction-related hospitalisations: a population-based cohort study. Pharmacoepidemiol Drug Saf. 2008;17:365-71.

9. Lazarou J, Pomeranz BH, Corey PN. Incidence of adverse drug reactions in hospitalized patients: a meta-analysis of prospective studies. JAMA. 1998;279:1200-5.

10. Wester K, Jönsson AK, Spigset $O$, Druid $H$, Hägg $S$. Incidence of fatal adverse drug reactions: a population based study. $\mathrm{Br} \mathrm{J}$ Clin Pharmacol. 2008;65:573-9.

11. Pardo Cabello AJ, González Contreras LG, Manzano Gamero MV, Gómez Jiménez FJ, Puche Cañas E. Prevalence of fatal adverse drug reactions in hospitalized patients. Int $\mathrm{J}$ Clin Pharmacol Ther. 2009;47:596-602.

12. Rawlins MD. The challenge to pharmacoepidemiology. Pharmacoepidemiol Drug Saf. 1995;4:5-9.

13. Herdeiro MT, Ferreira M, Ribeiro-Vaz I, Junqueira Polónia J, CostaPereira A. O sistema português de farmacovigilância. Acta Med Port. 2012;25:241-9.

\section{CONCLUSÕES}

Os profissionais de saúde da região Centro de Portugal têm vindo a aumentar o seu contributo para a produção de novo conhecimento acerca da segurança de medicamentos. Este facto sugere a importância e a relevância crescente da atividade da UFC, que verificou um aumento da taxa de notificação global e de notificação de reações adversas simultaneamente graves e desconhecidas. O aumento da taxa de notificação espontânea, através de uma maior adesão dos profissionais de saúde a este método de monitorização da segurança dos medicamentos na prática clínica, permitirá produzir mais conhecimento cuja consequência será a melhor avaliação das relações benefício/ risco dos medicamentos e a maior segurança dos doentes.

\section{CONFLITOS DE INTERESSE}

Os autores declaram não ter qualquer conflito de interesse relativamente ao presente artigo.

\section{FONTES DE FINANCIAMENTO}

Os autores declaram não ter recebido qualquer subsídio relativo ao presente artigo.

14. Directiva 2010/84/EU do Parlamento Europeu e do Conselho de 15 de Dezembro de 2010 que altera, no que diz respeito à farmacovigilância, a Directiva 2001/83/CE que estabelece um código comunitário relativo aos medicamentos para uso humano. Jornal oficial L348, 31/12/2010, p.74-99; [consultado 2014 Jul 14]. Disponível em: http://ec.europa.eu/ health/files/eudralex/vol-1/dir_2010_84/dir_2010_84_en.pdf.

15. Cabrita da Silva J, Soares MA, Martins SO. Reações adversas a medicamentos: Análise da base de dados do Sistema Nacional de Farmacovigilância (SVIG) 2009-2011. Relatório Final. (2012); [consultado 2014 Jul 22]. Disponível em: https://www.infarmed.pt/ portal/page/portal/INFARMED/MEDICAMENTOS_USO_HUMANO/ FARMACOVIGILANCIA/Relatorio_analise_dados_SVIG_2009_2011. pdf.

16. Jones J.Assessing causality of case reports of suspected adverse events In: Strom B, Kimmel S, Hennessy S, editors. Pharmacoepidemiology. $5^{\text {th }}$ ed. Chichester: John Wiley \& Sons; 2012. p. 583-600.

17. Instituto Nacional de Estatística. Anuário Estatístico da Região Centro 2012. (2013); [consultado 2014 Jul 29]. Disponível em: http://www.ine.pt/ xportal/xmain?xpid=INE\&xpgid=ine_publicacoes\&PUBLICACOESpub_ boui=209571958\&PUBLICACOESmodo=2.

18. WHO Collaboration Centre for Drug Statistics Methodology. ATC/DDD Index 2014; [consultado 2014 Jul 22]. Disponível em: http://www.whocc. no/atc_ddd_index/.

19. Mendes D, Alves C, Batel-Marques F. Safety of biologics approved for treating rheumatoid arthritis: analysis of spontaneous reports of adverse events. Clin Rheumatol. 2013;32:1207-14.

20. Mendes D, Alves C, Batel-Marques F. Safety profiles of adalimumab, etanercept and infliximab: a pharmacovigilance study using a measure of disproportionality in a database of spontaneously reported adverse events. J Clin Pharm Ther. 2014;39:307-13.

21. Alves C, Batel-Marques F, Macedo AF. Data sources on drug safety evaluation: a review of recent published meta-analyses. Pharmacoepidemiol Drug Saf. 2012;21:21-33.

22. International Conference on Harmonisation of Technical Requirements for Registration of Pharmaceuticals for Human Use (ICH). Medical Dictionary for Regulatory Activities (MedDRA); [consultado 2014 Jul 22] Disponivel em: http://www.ich.org/products/meddra.html.

23. $\mathrm{ICH}$ Harmonized Tripartite Guideline. Clinical safety data management: definitions and standards for expedited reporting E2A. International conference on harmonisation of technical requirements for registration of pharmaceuticals for human use. 1994; [consultado 2014 Jul 22]. Disponivel em: http://www.ich.org/fileadmin/Public_Web_Site/lCH_ 
Products/Guidelines/Efficacy/E2A/Step4/E2A Guideline.pdf.

24. Mendes D, Alves C, Batel Marques F. latrogenia grave desconhecida, notificações e notificadores: Resultados da actividade da Unidade de Farmacovigilância do Centro. Rev Port Med Geral Fam. 2012;28:34-40.

25. Hill BA. The environment and disease: Association or causation? Proc R Soc Med. 1965; 58: 295-300.

26. Agbabiaka TB, Savovíc J, Ernst E. Methods for causality assessment of adverse drug reactions: a systematic review. Drug Saf. 2008;31:21-37.

27. Meyboom RH, Hekster YA, Egber AC, Gribnau FW, Edwards IR. Causal or casual? The role of causality assessment in pharmacovigilance. Drug Saf. 1997;17:374-89.

28. Goldman SA. Limitations and strengths of spontaneous reports data. Clin Ther. 1998;20:C40-4.

29. Hartmann K, Doser AK, Kuhn M. Postmarketing safety information: how useful are spontaneous reports? Pharmacoepidemiol Drug Saf. 1999;8:S65-71.

30. World Health Organization (WHO), Uppsala Monitoring Centre. Reporting trends; [consultado 2014 Jul 22]. Disponível em: http://whoumc.org/DynPage. aspx?id=108476\&mn1=7347\&mn2=7252\&mn3=732 $2 \& m n 4=7558$.

31. Kopečná E, Deščíková V, Vlček J, Mladá J. Adverse drug reaction reporting in the Czech Republic 2005-2009. Int J Clin Pharm. 2011;33:683-9.

32. Marques J, Ribeiro-Vaz I, Pereira AC, Polónia J. A survey of spontaneous reporting of adverse drug reactions in 10 years of activity in a pharmacovigilance centre in Portugal. Int J Pharm Pract. 2014;22:27582.

33. Scicchitano F, Giofrè C, Palleria C, Mazzitello C, Ciriaco M, Gallelli L, et al. Pharmacovigilance and drug safety 2011 in Calabria (Italy): adverse events analysis. J Res Med Sci. 2012;17:872-5.

34. Giofrè C, Scicchitano F, Palleria C, Mazzitello C, Ciriaco M, Gallelli L, et al. Pharmacovigilance and drug safety in Calabria (Italy): 2012 adverse events analysis. J Pharmacol Pharmacother. 2013;4:S55-60.

35. Mendes D, Alves C, Batel Marques F. Nurses' spontaneous reporting of adverse drug reactions: expert review of routine reports. J Nurs Manag. 2014;22:322-30.

36. Bäckström $M$, Mjörndal $T$, Dahlqvist $R$. Spontaneous reporting of adverse drug reactions by nurses. Pharmacoepidemiol Drug Saf. 2002;11:647-50.

37. Hall M, McCormack P, Arthurs N, Feely J. The spontaneous reporting of adverse drug reactions by nurses. Br J Clin Pharmacol. 1995;40:173-5.

38. Morrison-Griffiths S, Walley TJ, Park BK, Breckenridge AM, Pirmohamed M. Reporting of adverse drug reactions by nurses. Lancet. 2003;361:1347-8

39. Ulfvarson J, Mejyr S, Bergman U. Nurses are increasingly involved in pharmacovigilance in Sweden. Pharmacoepidemiol Drug Saf. 2007;16:532-7.

40. Guerra LN, Clérigo MI. Relatório anual 2013 - Notificações e Casos de RAM recebidos no SNF (Sistema Nacional de Farmacovigilância). Infarmed (2013); [consultado em 2014 Dez 16]. Disponível em http:// www.infarmed.pt/portal/page/portal/INFARMED/MEDICAMENTOS_ USO_HUMANO/FARMACOVIGILANCIA/NOTIFICACAO_DE_RAM/ RELATORIOS_ANALISE_PERIODICA_DADOS/Relatorio\%20anual. pdf.

41. Blenkinsopp A, Wilkie P, Wang M, Routledge PA. Patient reporting of suspected adverse drug reactions: a review of published literature and international experience. Br J Clin Pharmacol. 2007;63:148-56.

42. de Langen J, van Hunsel F, Passier A, de Jong-van den Berg L, van Grootheest K. Adverse drug reaction reporting by patients in the Netherlands: three years of experience. Drug Saf. 2008;31:515-24.
43. Mitchell AS, Henry DA, Sanson-Fisher R, O'Connell DL. Patients as a direct source of information on adverse drug reactions. BMJ. 1988;297:891-3.

44. Rolfes L, Wilkes S, van Hunsel F, van Puijenbroek E, van Grootheest K. Important information regarding reporting of adverse drug reactions: a qualitative study. Int J Pharm Pract. 2014;22:231-3.

45. Hazell L, Shakir SA. Under-reporting of adverse drug reactions: a systematic review. Drug Saf. 2006;29:385-96.

46. Vallano A, Cereza G, Pedròs C, Agustí A, Danés I, Aguilera C, et al. Obstacles and solutions for spontaneous reporting of adverse drug reactions in the hospital. Br J Clin Pharmacol. 2005;60:653-8.

47. Ferreira M, Rodrigues F, Brown K, Palminha P, Cordeiro E. Surto de parotidite epidémica em população com elevada taxa de vacinação: 2012-2013. Acta Pediátr Port. 2013;44:S39.

48. Baniasadi S, Fahimi F, Shalviri G. Developing an adverse drug reaction reporting system at a teaching hospital. Basic Clin Pharmacol Toxicol. 2008;102:408-11.

49. Alves $C$, Macedo AF, Batel Marques F. Sources of information used by regulatory agencies on the generation of drug safety alerts. Eur $\mathrm{J}$ Clin Pharmacol. 2013;69:2083-94.

50. Arnott J, Hesselgreaves H, Nunn AJ, Peak M, Pirmohamed M, Smyth $\mathrm{RL}$, et al. Enhancing communication about paediatric medicines: lessons from a qualitative study of parents' experiences of their child's suspected adverse drug reaction. PLoS One. 2012;7:e46022.

51. Barata I, Alves C, Mendes D, Fontes Ribeiro C, Batel Marques F. The preventability of adverse drug reactions occuring with antineoplastic drugs - an evaluation of spontaneously reported cases. Drug Saf. 2013;36:831.

52. Suh DC, Woodall BS, Shin SK, Hermes-De Santis ER. Clinical and economic impact of adverse drug reactions in hospitalized patients. Ann Pharmacother. 2000;34:1373-9.

53. Arnaiz JA, Carné X, Riba N, Codina C, Ribas J, Trilla A. The use of evidence in pharmacovigilance. Case reports as the reference source for drug withdrawals. Eur J Clin Pharmacol. 2001;57:89-91.

54. Wysowski DK, Swartz L. Adverse drug event surveillance and drug withdrawals in the United States, 1969-2002: the importance of reporting suspected reactions. Arch Intern Med. 2005;165:1363-29.

55. Olivier P, Montastruc JL. The nature of the scientific evidence leading to drug withdrawals for pharmacovigilance reasons in France. Pharmacoepidemiol Drug Saf. 2006;15:808-12.

56. Clarke A, Deeks JJ, Shakir SA. An assessment of the publicly disseminated evidence of safety used in decisions to withdraw medicinal products from the UK and US markets. Drug Saf. 2006;29:175-81.

57. Paludetto MN, Olivier-Abbal $P$, Montastruc $\mathrm{JL}$. Is spontaneous reporting always the most important information supporting drug withdrawals for pharmacovigilance reasons in France? Pharmacoepidemiol Drug Saf. 2012;21:1289-94.

58. McNaughton R, Huet G, Shakir S. An investigation into drug products withdrawn from the EU market between 2002 and 2011 for safety reasons and the evidence used to support the decision-making. BMJ Open. 2014;4:e004221.

59. Palleria C, Leporini C, Chimirri S, Marrazzo G, Sacchetta S, Bruno $L$, et al. Limitations and obstacles of the spontaneous adverse drugs reactions reporting: Two "challenging" case reports. J Pharmacol Pharmacother. 2013;4:S66-72.

60. González-Rubio F, Calderón-Larrañaga A, Poblador-Plou B, NavarroPemán C, López-Cabañas A, Prados-Torres A. Underreporting of recognized adverse drug reactions by primary care physicians: an exploratory study. Pharmacoepidemiol Drug Saf. 2011;20:1287-94. 
Francisco BATEL-MARQUES, Diogo MENDES, Carlos ALVES, Ana PENEDONES, Patricia DIAS, Angelina MARTINS, Luiz Miguel SANTIAGO, Carlos FONTES-RIBEIRO, Margarida CARAMONA, Tice MACEDO

\section{Farmacovigilância em Portugal: Atividade da Unidade Regional do Centro}

Acta Med Port 2015:28:222-232

Publicado pela Acta Médica Portuguesa, a Revista Científica da Ordem dos Médicos

Av. Almirante Gago Coutinho, 151

1749-084 Lisboa, Portugal.

Tel: +351218428215

E-mail: submissao@actamedicaportuguesa.com

www.actamedicaportuguesa.com

ISSN:0870-399X | e-ISSN: 1646-0758

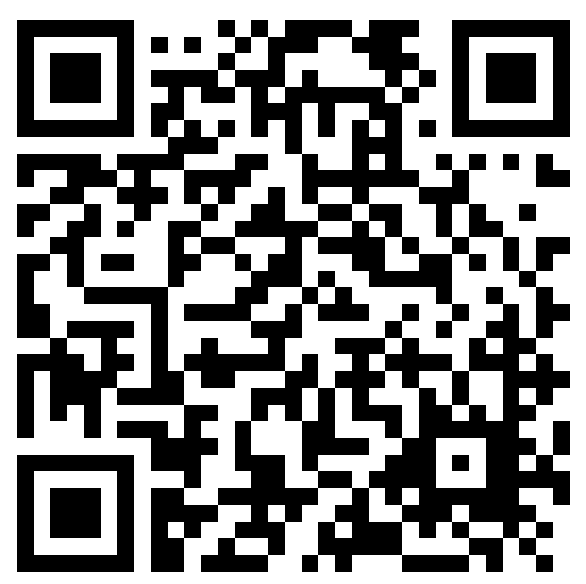

